\title{
Tensile stress generation by optical breakdown in tissue: Experimental investigations and numerical simulations
}

\author{
Alfred Vogel ${ }^{1}$, Richard J. Scammon ${ }^{2}$, and Robert P. Godwin ${ }^{3}$ \\ 1) Medizinisches Laserzentrum Lübeck, D-23562 Lübeck, Germany \\ 2) Los Alamos National Laboratory, ESA-EA, Los Alamos, NM 87545 \\ 3) Los Alamos National Laboratory, X-NH, Los Alamos, NM 87545 in \\ MAR 12 ISPS
}

ABSTRACT

Biological tissue is more susceptible to damage from tensile stress than to compressive stress. Tensile stress may arise through the thermoelastic response of laser-irradiated media. Optical breakdown, however, has to date been exclu-sively associated with compressive stress. We show that this is appropriate for water, but not for tissues for which the elasticplastic material response needs to be considered.

The acoustic transients following optical breakdown in water and cornea were measured with a fast hydrophone and the cavitation bubble dynamics, which is closely linked to the stress wave generation, was documented by flash photography. Breakdown in water produced a monopolar acoustic signal and a bubble oscillation in which the expansion and collapse phases were symmetric. Breakdown in comea produced a bipolar acoustic signal coupled with a pronounced shortening of the bubble expansion phase and a considerable prolongation of its collapse phase. The tensile stress wave is related to the abrupt end of the bubble expansion.

Numerical simulations using the MESA-2D code were performed assuming elastic-plastic material behavior in a wide range of values for the shear modulus and yield strength. The calculations revealed that consideration of the elastic-plastic material response is essential to reproduce the experimentally observed bipolar stress waves. The tensile stress evolves during the outward propagation of the acoustic transient and reaches an amplitude of $30-40 \%$ of the compressive pulse.

Keywords: Laser-induced breakdown, stress waves, cavitation, cornea, tensile stress, tissue damage, elastic-plastic response.

\section{INTRODUCTION}

Biological tissue is more susceptible to damage from tensile stress than to compressive stress ${ }^{1,2}$. An understanding of material failure and collateral damage during pulsed laser surgery therefore requires knowledge of the mechanisms of tensile stress generation. Tensile stress may arise through the reflection of a compressive stress wave at the boundary to a medium with low acoustic impedance, during surface ablation under stress confinement conditions ${ }^{3}$, or through the thermoelastic response of laser-irradiated spatially limited absorbers in an acoustically homogeneous medium ${ }^{4.5}$. In contrast to thermoelastic mechanisms, optical breakdown has to date been exclusively associated with compressive stress ${ }^{6.7}$. We show that this is appropriate for water, but not for optical breakdown in tissues exhibiting viscoelastic material properties.

Stress wave generation is closely related to the formation of cavitation bubbles; because they are both driven by the expansion of the heated material in the breakdown volume. Modifications of the stress wave emission due to the viscoelastic tissue properties will therefore be linked to changes in the bubble dynamics. In this study, we investigate the bubble dynamics and stress wave emission in corneal tissue both experimentally and by numerical simulations. We selected this example, because the corneal transparency allows observation and documentation of the bubble dynamics by high speed photography. An analysis of cavitation and stress wave emission in corneal tissue is, furthermore, clinically relevant for intrastromal refractive surgery with ultrashort laser pulses ${ }^{8.9}$. The main aim of our study, however, is to achieve a general

Correspondence: Alfred Vogel, PhD, Medical Laser Center Lübeck, Peter-Monnik-Weg 4, D-23562 Lübeck, Germany.

FAX: x49-451-505 486; e-mail: vogel@mll.mu-luebeck.de

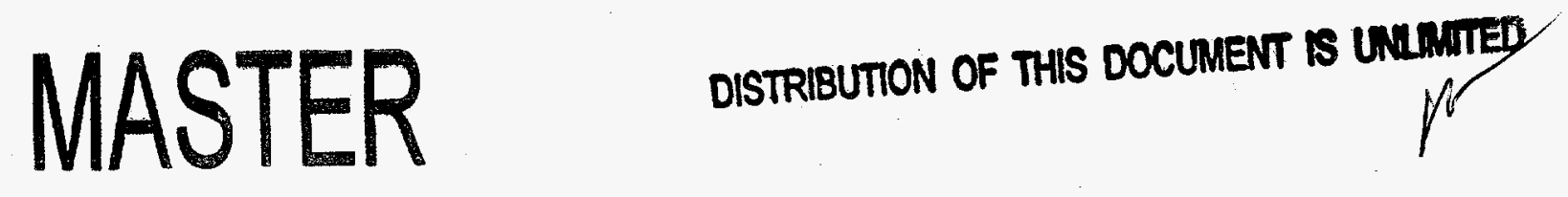


understanding of cavitation bubble dynamics and stress wave generation in tissues rather than to model one specific clinical application.

The numerical simulations were performed by using the MESA-2D code assuming elastic-perfectly-plastic tissue behavior (neglecting viscosity). The elastic-plastic material behavior has only recently been taken into account for the modeling of laser-tissue interactions. Glinsky et al. ${ }^{10}$ introduced an extended Rayleigh model of bubble evolution with material strength and compared it to the results of detailed dynamic simulations, and Chapyak and Godwin " showed that consideration of the elastic-plastic tissue response is essential for an understanding of the ablation mechanisms in laser thrombolysis.

\section{MATERIALS AND METHODS}

\subsection{Experiments}

The experiments were performed on cornea specimens from sheep eyes obtained from a slaughterhouse. The specimens were used within 6 hours after enucleation and kept in a refrigerator until use. They were mounted on a teflon holder and immersed in a cuvette filled with physiological saline. To get smooth plane cuts, the corneal excisions were performed with a preparation blade. Nd:YAG laser pulses $(1064 \mathrm{~nm})$ with $30 \mathrm{ps}$ duration and $300 \mu \mathrm{J}$ pulse energy were focused through a contact lens into the corneal stroma (focusing angle $18^{\circ}$ ). The contact lens was built into the cuvette wall to avoid spherical aberrations ${ }^{12}$. The light was incident from the epithelial side. Photographs were taken through a side of the corneal specimen such that the pictures showed a cross sectional view of the cornea and a side view of the cavitation bubbles. To minimize edematous changes of the corneal stroma, the experiments were performed within 10 minutes of excision of the specimen. For comparison, the bubble dynamics and acoustic emission in saline and water were also documented. The sequence of events in physiological saline is very similar to the dynamics in water, because the optical breakdown thresholds in both liquids are almost the same ${ }^{12,13}$.

The cavitation bubble dynamics was recorded by time-resolved flash photography with 20 ns exposure time ${ }^{12}$ (Nanolite KL-L, High Speed Photo-Systeme, Wedel, Germany). The bubbles were transilluminated with collimated light, and photographs were taken with $7 x$ magnification on Kodak T Max 100 film using a Leitz Photar lens $(F=3.5, f=40 \mathrm{~mm}$ ). The time between the Nd:YAG laser pulse producing breakdown and the flash could be adjusted in steps of $1 \mu$ starting from a minimum delay of $3 \mu$ shich was given by the electronic transition time of the Nanolite driver.

The acoustic transients emitted after optical breakdown in water and cornea were measured at $10 \mathrm{~mm}$ distance from the breakdown site using a PVDF hydrophone of 12 ns rise time (CERAM miniature hydrophone, Lund, Sweden). Each measurement was first performed for optical breakdown in the cornea specimen. Aftervards, the specimen was removed without changing the location of the hydrophone, and the experiment was repeated for optical breakdown in saline.

\subsection{Material Parameters of Corneal Tissue}

When subjected to small and moderate stresses, tissue exhibits elastic behavior, whereas at stress amplitudes beyond the ultimate tensile stress it undergoes plastic deformation and fails. The material response in the elastic domain is characterized by the elastic modulus $E=Y / \varepsilon$ where $Y$ and $\varepsilon$ denote stress and strain, respectively. The stress-strain relation of most soft tissues can be characterized by three regions (Fig. 1). At low stress there is a region (1) of relatively low elastic modulus in which large extensions may occur for small increases in stress. At high stresses below the ultimate tensile strength of the tissue, there is a region (2) of high elastic modulus in which extensions are much smaller for a given stress increment. The elastic properties in both regions are approximately linear in many tissues and, in principle, one can derive an elastic modulus from the slope of the stress-strain response in either of these quasi-linear regions ${ }^{2}$. Beyond a certain stress level (ultimate tensile strength $Y_{0}$ ), the tissue undergoes plastic deformation where a large strain is produced by a constant stress level (3), and after material failure, additional strain may be produced with stress values smaller than $Y_{0}(4)$.

Region (1) usually applies to physiological conditions - in cornea, for example, to an intraocular pressure of $15 \mathrm{~mm} \mathrm{Hg}$. Measurement values for the elastic modulus in this region obtained by uniaxial tensile loading of cornea strips range from 3.4 bar to $5 \mathrm{bar}^{14}$. Measurements performed in region (2) at a stress level of $2350 \mathrm{~mm} \mathrm{Hg} \mathrm{(3.1} \mathrm{bar),} \mathrm{however,} \mathrm{yielded} \mathrm{values}$ between 210 bar and $570 \mathrm{bar}^{14}$. The same trend was observed when the mechanical performance was studied by pressure - 


\section{DISCLAIMER}

This report was prepared as an account of work sponsored by an agency of the United States Government. Neither the United States Governmeat aor any agency thereof, nor any of their employees, makes any warranty, express or implied, or assumes any legai liability or responsibility for the accuracy, completeness, or usefulness of any information, apparatus, product, or process disclosed, or represents that its use would not infringe privately owned rights. Reference berein to any specific commercial product, process, or service by trade name, trademark, inanufacturer, or otherwise does not necessarily constitute or imply its endorsement, recommendation, or favoring by the United States Government or any ageacy thereof. The views and opinions of authors expressed herein do not necessarily state or reflect those of the United States Goverwment or any agency thereof. 


\section{DISCLAIMER}

\section{Portions of this document may be illegible in electronic image products. Images are produced from the best available original document.}




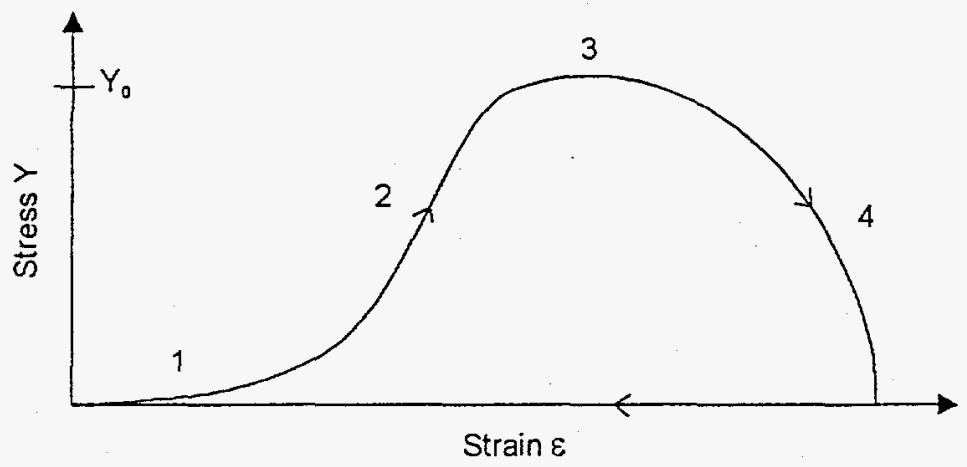

Figure 1: Schematic representation of the stress-strain relationship for soft tissues, including the region of material failure.

loading of intact eyes: here the elastic modulus increased from 30 bar at $2-10 \mathrm{~mm} \mathrm{Hg}$ to $200 \mathrm{bar}$ at $25-100 \mathrm{~mm} \mathrm{Hg}^{15}$. The strong nonlinearity of the stress-strain relationship is not a unique feature of corneal tissue. The elastic modulus of arterial walls associated with normal physiological conditions, for example, is 10-20 bar, but at blood pressures in excess of $200 \mathrm{~mm}$ $\mathrm{Hg}$ the modulus reaches $200 \mathrm{bar}^{2}$.

The nonlinearity of the stress-strain relationship requires great care in making the parameter choice when the mechanical response is simulated through experiments with tissue phantoms or in numerical calculations. In some earlier studies, values of $\approx 10$ bar were quoted for the elastic modulus of tissue ${ }^{16,17}$. These values apply to region (1) of the stressstrain relationship, and are obviously too low to describe the tissue response during pulsed laser ablation or optical breakdown, respectively, where pressures in the range of hundreds of bars to several kilobars are reached ${ }^{6.18}$.

A further complication arises through the fact that most values for the elastic modulus are determined under static load or very low deformation rates, whereas the deformation rate during the bubble expansion in tissue is very high. Soergel et al. ${ }^{19}$ showed through dynamic mechanical analysis that the shear modulus of cornea measured in the direction parallel to the corneal surface is 15 times higher at an excitation frequency of $1 \mathrm{MHz}$ than at $1 \mathrm{~Hz}\left(G_{=}=2\right.$ bar at $1 \mathrm{MHz}$ ). Similar considerations may apply for the ultimate tensile strength $Y_{0}$ (i.e. the plastic flow stress) of tissue which is usually measured under static load, but could be higher at high deformation rates where the tissue is stiffer. Values for the ultimate tensile strength determined under static load range from $34 \mathrm{bar}^{2}$ through $125 \mathrm{bar}^{20}$ to $150 \mathrm{bar}^{21}$.

All material parameters quoted above refer to deformations parallel to the corneal surface. The cornea is highly nonisotropic because of its lamellar structure and the directional orientation of the collagen fibrils within each lamella ${ }^{22}$. The parameters for deformations perpendicular to the surface will therefore differ from those for in-plane deformations. In isotropic media, the elastic modulus $E$ and the shear modulus $G$ are related by

$$
E=2(1+\mu) G,
$$

where $\mu \approx 0.5$ is the ratio between lateral contraction and axial strain. We therefore obtain $E \approx 3 G$. The lamellar structure of the comea leads to deviations from this approximate relation for isotropic materials: Soergel et al. ${ }^{19}$ found $E_{1} \approx 100 G_{=}$ for the relation between the elastic modulus perpendicular to the corneal surface and the shear modulus parallel to the surface (i.e. the cornea is easy to shear but hard to compress), and Chang et al. ${ }^{23}$ obtained $E_{=} \approx 100 G_{=}$. These ratios apply to measurements on whole corneas in which the lamellae can easily slide over one another. The 'microscopic' shear modulus for forces acting only within individual lamellae has not yet been measured, but will certainly be much larger than the 'macroscopic' value of $G_{m}$. The value of $G_{\perp}$ has not yet been measured, and will probably also be larger than the 'macroscopic' value of $G_{=}$. For deformations, where the lamellae are not sheared with respect to one another, one may therefore be able to use the relation $E \approx 3 G$ for isotropic media as a first approximation.

When a cavitation bubble is produced inside the tissue, the tissue response is chracterized by different regions of the stress-strain curve (Fig 1), depending on the distance from the bubble center and the time during bubble oscillation. The material parameters used for numerical simulations of the bubble dynamics and stress wave generation can therefore only be rough estimates, and the assumption of fixed values for these parameters is only a simple approximation. The above review of corneal material properties allow to estimate, nevertheless, the order of magnitude for meaningful values of the elastic 
modulus and yield strength: $E$ should be about $500-600$ bar, but might be considerably higher in the early phase of bubble expansion when the bubble pressure has dropped just below the plastic flow stress of the tissue. The plastic flow stress $Y_{0}$ should be about 100 bar or larger.

\subsection{Numerical Simulations}

The nominal geometry used in the MESA-2D 24 numerical simulations is presented in Fig. 2 . The two-dimensional calculations were run with cylindrical geometry and a mesh resolution fine enough to resolve the stress waves within the volume of interest. Check runs were used to verify that the mesh was satisfactory. The energy representing the laser radiation was deposited instantaneously at the start of the problem into a spherical energy deposition region of $20 \mu \mathrm{m}$ radius. An energy density of $7800 \mathrm{~J} / \mathrm{g}$ determined from photographs of the plasma size $\mathrm{s}^{25}$ and transmission measurements ${ }^{26}$ was used to simulate the $300 \mu \mathrm{J}, 30$ ps laser pulse. A one bar ambient pressure was applied to simulate atmospheric pressure. The pressure time edits along the $Z$-axis were taken at a radius of $0.02 \mathrm{~cm}$ to avoid numerical instabilities along the $r=0$ boundary.
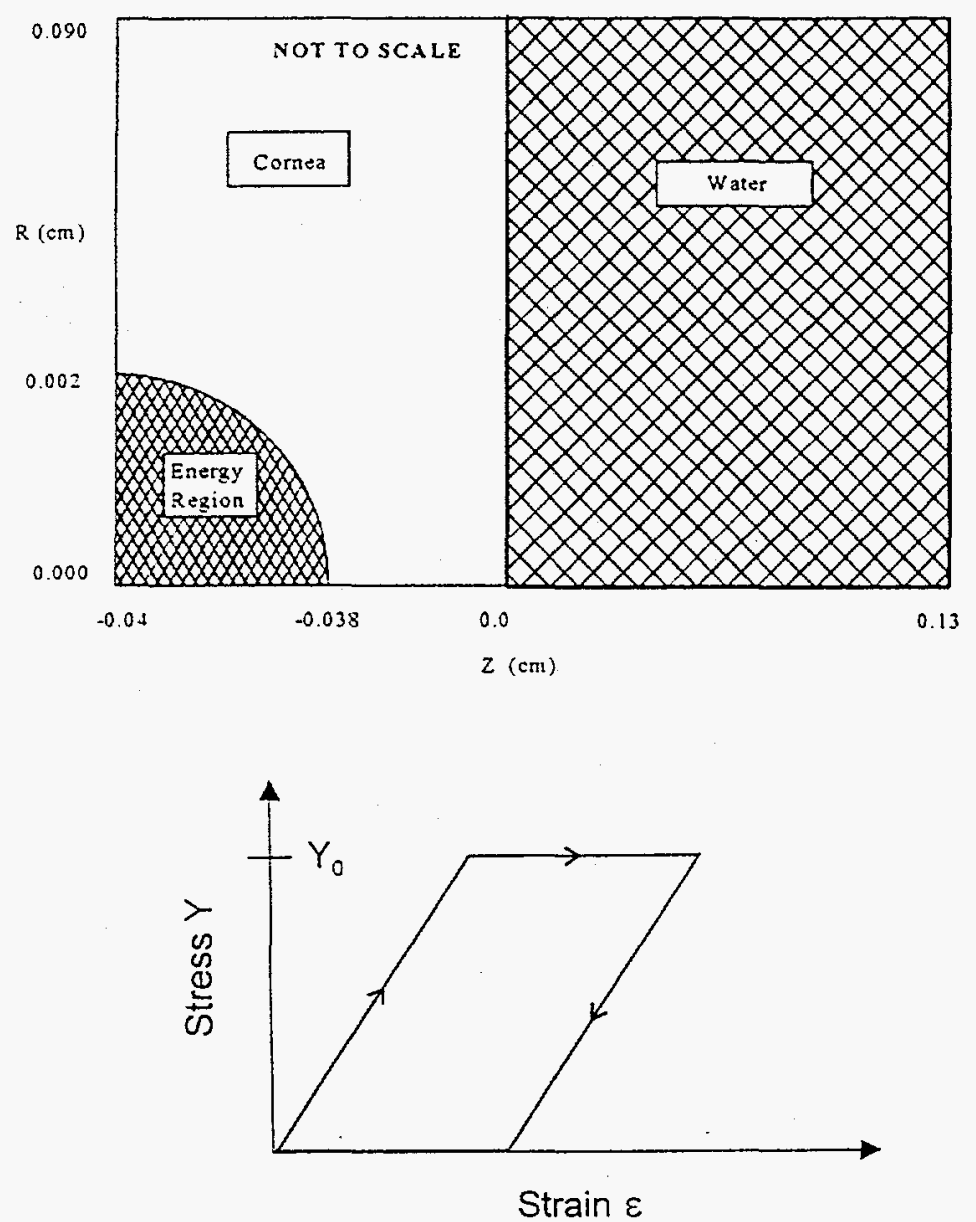

Figure 2: Geometry for the simulations of cavitation bubble dynamics and stress wave generation in coneal tissue. The energy deposition volume was assumed to be spherical, with a radius of $20 \mu \mathrm{m}$. An energy density of $7800 \mathrm{~J} / \mathrm{g}$ was used to simulate the laser pulse The tensile strength of water (cavitation threshold) was set to -10 bar. All outer boundaries are rigid.
Figure 3: Stress-strain behavior assumed for the simulations. For stress values below the plastic flow stress $Y_{0}$, the material behaves according to Hookes law, and for stresses above $Y_{0}$ it acts like a fluid. When the stress falls below $Y_{0}$, the material reacts elastic again. The relation between elastic modulus and shear modulus correponding to the linear part of the stress-strain curve is $E=2(1+\mu) \mathrm{G} \approx 3 G$.

The calculations used isotropic elastic-plastic material behavior for the cornea. A wide range of values for the shear modulus and yield strength were investigated. This partly reflects the uncertainty in the determination of the actual tissue parameters, but is also intended to yield an understanding of the parameter dependence of stress wave formation. The MESA-2D elastic-plastic model is a simple elastic-perfectly-plastic model with a constant yield surface. Fig. 3 shows the stress-strain relationship assumed for simulations. The J-shape of the actual stress-strain relation for soft tissues (see Fig. 1) and part 4 of the curve are not taken into account. We consider only the quasi-linear region (2) describing the response to moderate and high stresses. Once the ultimate tensile strength (plastic flow stress) of the tissue is exceeded, the material is 
assumed to behave perfectly plastic. There is no viscosity associated with the model. Possible changes in the shear modulus arising from material failure in the region surrounding the expanding cavitation bubble ${ }^{10}$ were not considered. The code uses the standard relation (Eq. 1) between shear and elastic modulus for isotropic media. Poisson's ratio $\mu$ was obtained from the SESAME equation of state table for water. It is 0.495 for $G=200$ bar, and 0.456 for $G=2000$ bar.

The tensile strength of the water or saline, respectively, is given by the cavitation threshold. The threshold for ultrasonic cavitation in tap water is -1 to -10 bar depending on the sound frequency ${ }^{27}$. The shorter the duration of the tensile stress during each cycle is, the higher is the tensile stress amplitude required to cause cavitation. The threshold for cavitation induced by single pressure transients of submicrosecond duration was determined by Paltauf et al. ${ }^{28}$ and Jacques et al. ${ }^{29}$ to be -7.5 to -8 bar. Because the tensile stress transients observed in our study had a duration of only about 70 ns FWHM (see below, Fig. 6), we assumed a cavitation threshold of -10 bar for the simulations.

\section{RESULTS}

\subsection{Experiments}

The cavitation bubble oscillation after optical breakdown in comea and water is shown in Figs. 4 and 5 , and the corresponding acoustic signals are presented in Fig. 6.

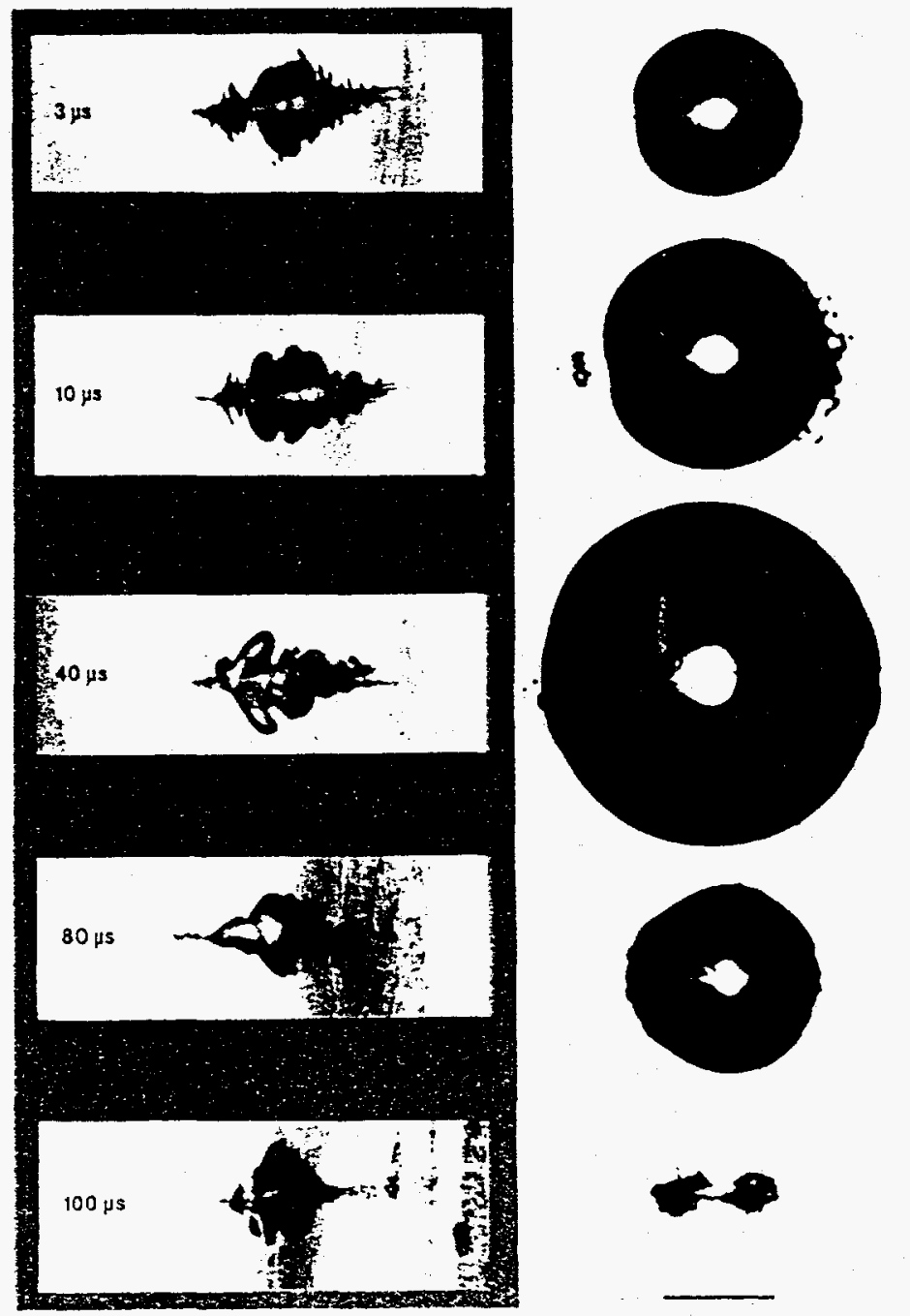

Figure 4: Cavitation bubble dynamics in cornea induced by a $300 \mu \mathrm{J}$ laser pulse of 30 ps duration (left column), and the corresponding bubble shapes in physiological saline (right column). Both picture series are composed of photographs of different events taken with increasing time delay after the laser pulse. The laser light was incident from the right. The scale represents a length of $200 \mu \mathrm{m}$.

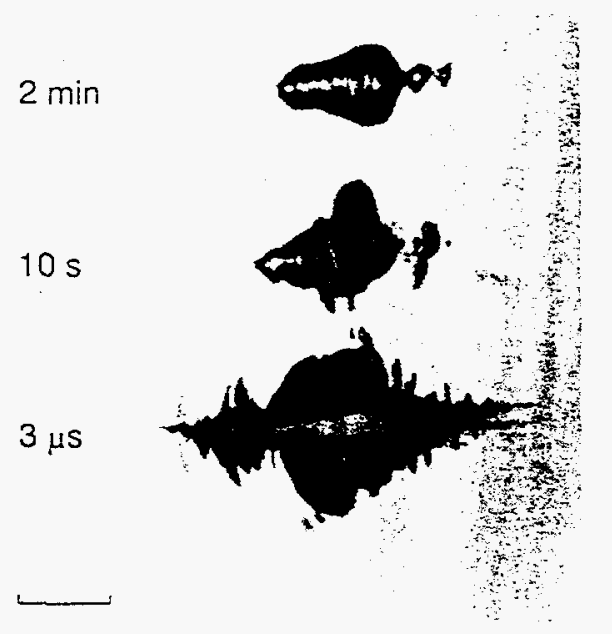

Figure 5: Cavitation bubbles produced by $300 \mu \mathrm{J}$ laser pulses focused in cornea, photographed at three different times after the laser pulse. The scale corresponds to a length of $100 \mathrm{um}$. 

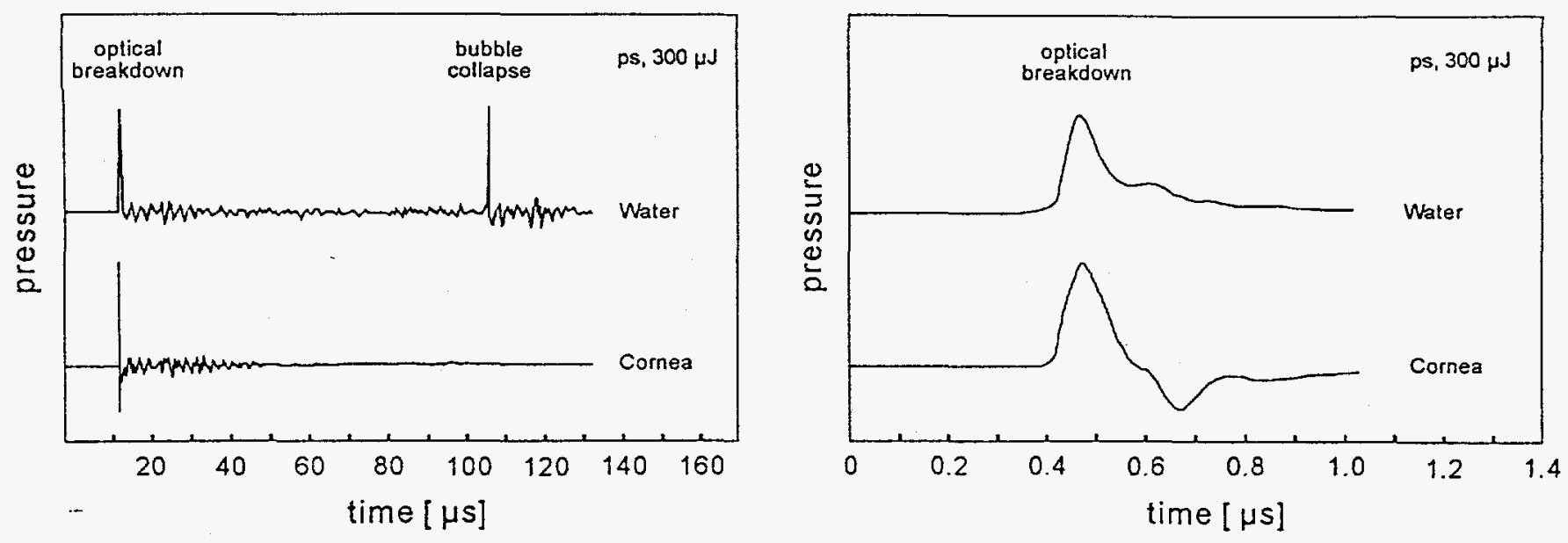

Figure 6: Acoustic emission after focusing of $300 \mu \mathrm{J}$ laser pulses in cornea and physiological saline, a) overview, b) detailed view of the breakdown pulse. The acoustic signals were measured at $10 \mathrm{~mm}$ distance from the breakdown site.

Optical breakdown in water produced a bubble oscillation with symmetric expansion and collapse phases (Fig. 4a). The corresponding acoustic signal was monopolar, and the pressure peaks from breakdown and bubble collapse had the same amplitude (Fig 6a).

When the breakdown occurred in corneal tissue, the bubble expansion phase was considerably shortened to a few microseconds, and the maximum bubble size strongly reduced (Fig. 4b). The collapse phase was prolonged to several minutes during which the gaseous bubble contents slowly diffused into the surrounding tissue (Fig. 5). Because of the strong damping of the bubble oscillation, no collapse pulse could be observed ${ }^{30}$ (Fig. 6a). The shortening of the bubble expansion phase was coupled with a change of the acoustic breakdown signal into a bipolar shape (Fig. 6b). The amplitude of the compressive pulse was the same as in water ( 2.4 bar at $10 \mathrm{~mm}$ distance from the source). The amplitude of the tensile stress wave $(-1.0$ bar) was about $40 \%$ of the compressive stress.

After the rapid expansion phase of the intrastromal cavitation bubble, Juhasz et al. ${ }^{31}$ observed slight oscillations of the bubble radius before a constant value was reached at 50-100 $\mu \mathrm{s}$. The oscillation period was 30-50 $\mu \mathrm{s}$, depending on the laser pulse energy. In our experiments, however, we did not observe similar bubble oscillations in corneal tissue (Fig. 4), possibly due to the limited temporal resolution of the photographic investigations.

\subsection{Numerical Simulations}

Figure 7 shows radius time curves for the initial phase of the bubble expansion in water and for several combinations of plastic flow stress $Y_{0}$ and shear modulus $G$. At the end of the bubble oscillations, a resonable agreement with the experimentally observed bubble radius of $\approx 85 \mu \mathrm{m}$ (Fig. 4) is achieved for $Y_{0}=100$ bar and $G=200$ bar (corresponding to $E=600 \mathrm{bar}$ ). These values were in section 2.2 presented as estimates of corneal tissue parameters for large deformation rates and stresses.

Stress signals in cornea and water for $Y_{0}=400$ bar and $G=200$ bar are shown in Fig. 8 . The first edit in cornea (Fig. $8 \mathrm{a},-0.03 \mathrm{~cm}$ ) shows the stress transient at a distance of $220 \mu \mathrm{m}$ from the laser focus and $300 \mu \mathrm{m}$ from the corneawater interface. Here, the stress wave consists of a short compressive pulse with an amplitude of $2.7 \mathrm{kbar}$ followed by a longer tensile stress wave with a mean amplitude of about -200 bar. The shape of the stress wave is obscured by oscillations with a period related to the sound transit time across the energy deposition region which are generated by shock relief of this volume. These oscillations are in part an artifact of the initial conditions in which deposition was modeled as an instantaneous step function jump from zero to full energy deposition at the border of the spherical energy deposition region. Close to the cornea-wvater interface, the mean tensile stress amplitude averaged over the oscillations is still about - 30 bar, and the amplitude of the compressive pulse is 320 bar (first edit in Fig $8 \mathrm{~b}$, at $-0.005 \mathrm{~cm}$ ). The ratio of tensile and compressive stress amplitudes increases slightly during stress wave propagation in the tissue and amounts to $\approx 0.1$ at the 


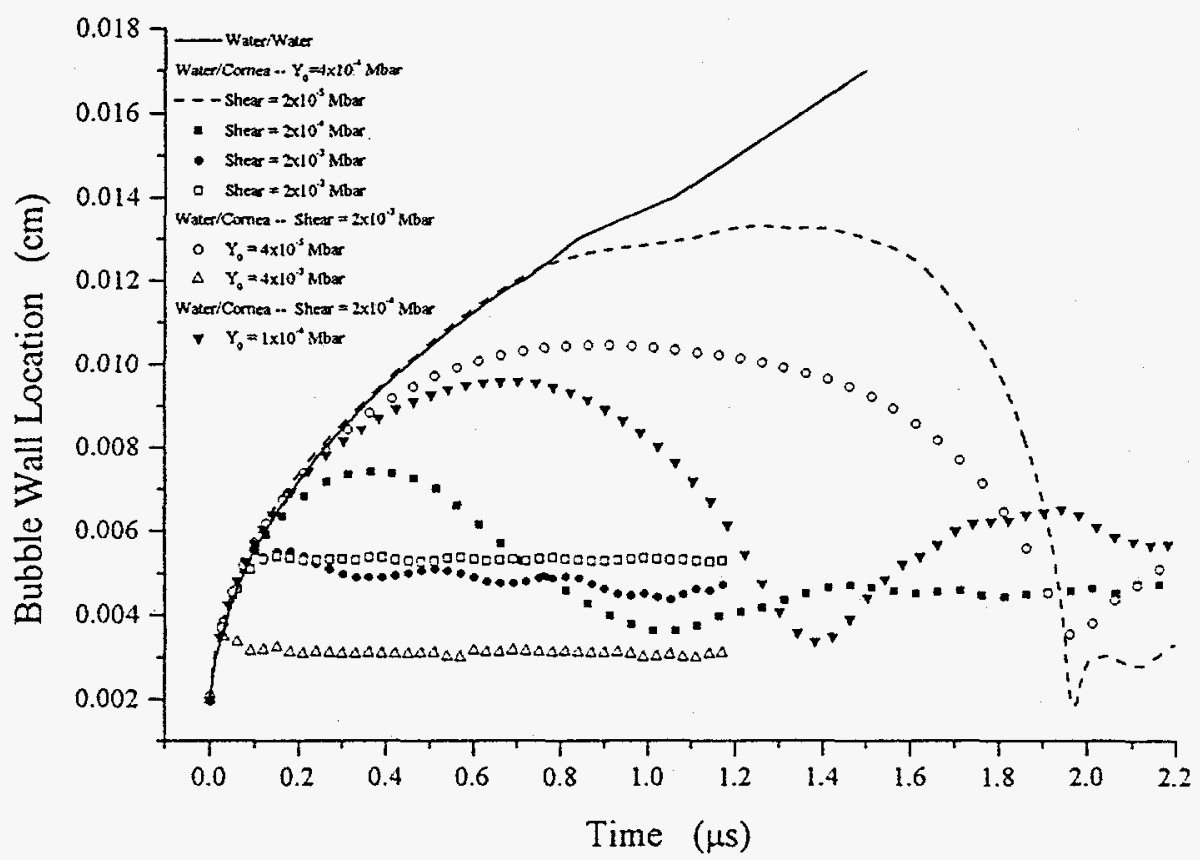

Figure 7: Radius time curves for the initial phase of the bubble expansion in water and for various combinations of plastic flow stress $Y_{0}$ and shear modulus $G$. The combination $Y_{0}=100 \mathrm{bar}, G=200$ bar yields reasonable agreement with the experimentally observed bubble size in figures 4 and 5 . For comparison: the values for PMMA are $Y_{0}=4200$ bar, and $G=23000$ bar.

cornea-ivater interface. When the stress wave enters the water, its tensile part is cut off at the cavitation threshold of - 10 bar. For some time, the amplitude of the tensile part remains constant, while the amplitude of the compressive part decreases due to the geometric attenuation of the spherical wave. After $1 \mathrm{~mm}$ propagation in water, the ratio between tensile stress and compressive stress is 0.2 . It is possible that the simulated ratio increases to 0.4 at $10 \mathrm{~mm}$ distance, as observed experimentally.

Figure 9 shows the evolution of the stress signals for considerably larger values of yield strength and shear modulus ( $Y_{0}=400$ bar, $G=2000$ bar). The qualitative picture is quite similar to that in Fig. 8, but the compressive amplitude at the cornea/water interface is smaller $(230 \mathrm{bar})$, and the tensile stress amplitude is aboutwice-as high $(\approx-45$ bar after averaging over the high frequency oscillations). The ratio between compressive and tensile stress is 0.2 at the cornea-water interface, and 0.22 after $1 \mathrm{~mm}$ propagation in water. The maximum tensile stress in cornea is -220 bar.

In order to compare the elastic-plastic model with other approaches to tissue response modeling, we also calculated the evolution of the bubble radius and the stress wave formation for an increased ambient pressure $p_{\infty}$ (Fig. 10). A maximum bubble radius of $85 \mu \mathrm{m}$ equal to the experimentally observed value is obtained for $p_{\infty}=250 \mathrm{bar}$, but unlike in the experiment, the bubble collapses again to a much smaller radius (Fig. 10a). Figure 10b demonstrates that only a rarefaction wave, but no no tensile stress wave is produced, again in contrast to the experiment.

Figure 11 shows an attempt to model the tissue response by varying the viscosity of the medium without considering its elasticity ${ }^{32}$. The experimentally observed bubble radius can be matched by setting the viscosity to $4.5 \mathrm{~Pa} \cdot \mathrm{s}$ (Fig. 11a). Compared to the results obtained using the elastic-plastic model, the stress amplitudes are then reduced by a factor of 10 at $z=-0.03$. The tensile stress amplitude is not larger than -10 bar inside the cornea, and is only -3 bar at the cornea-water interface (Fig. 11b). 
(a)

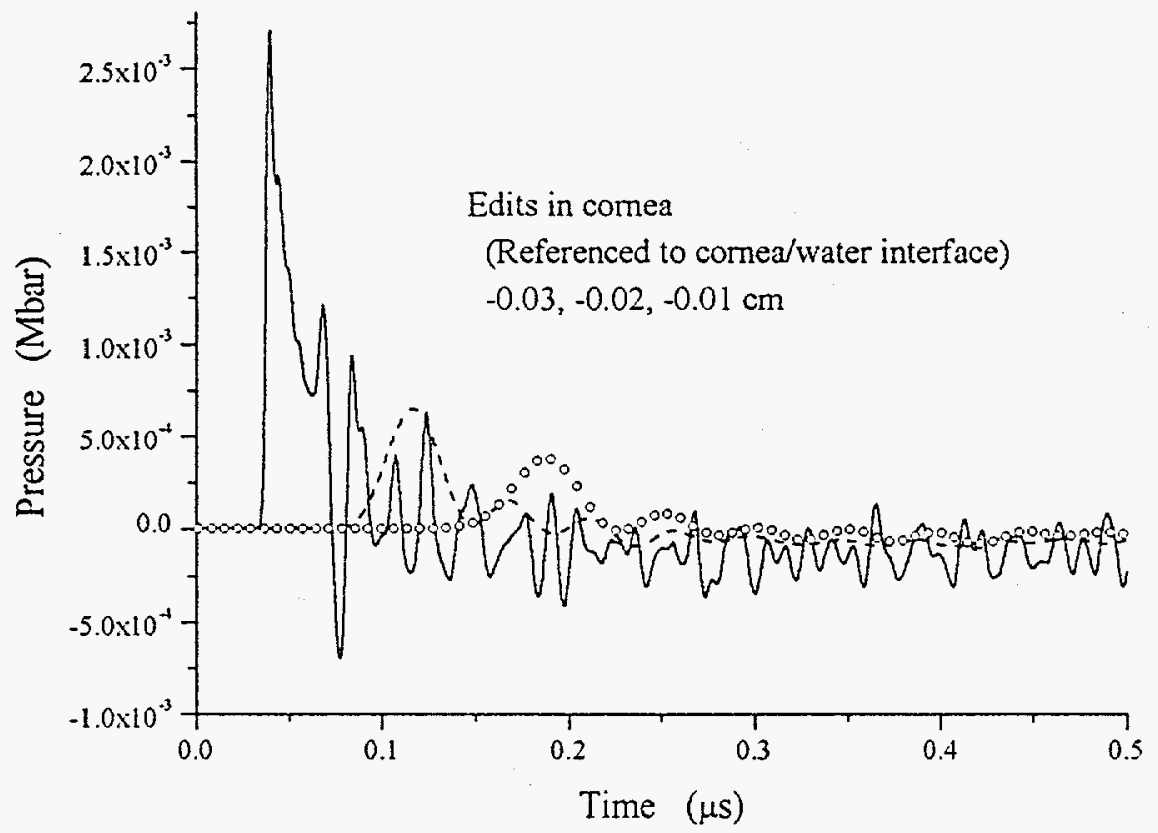

(b)

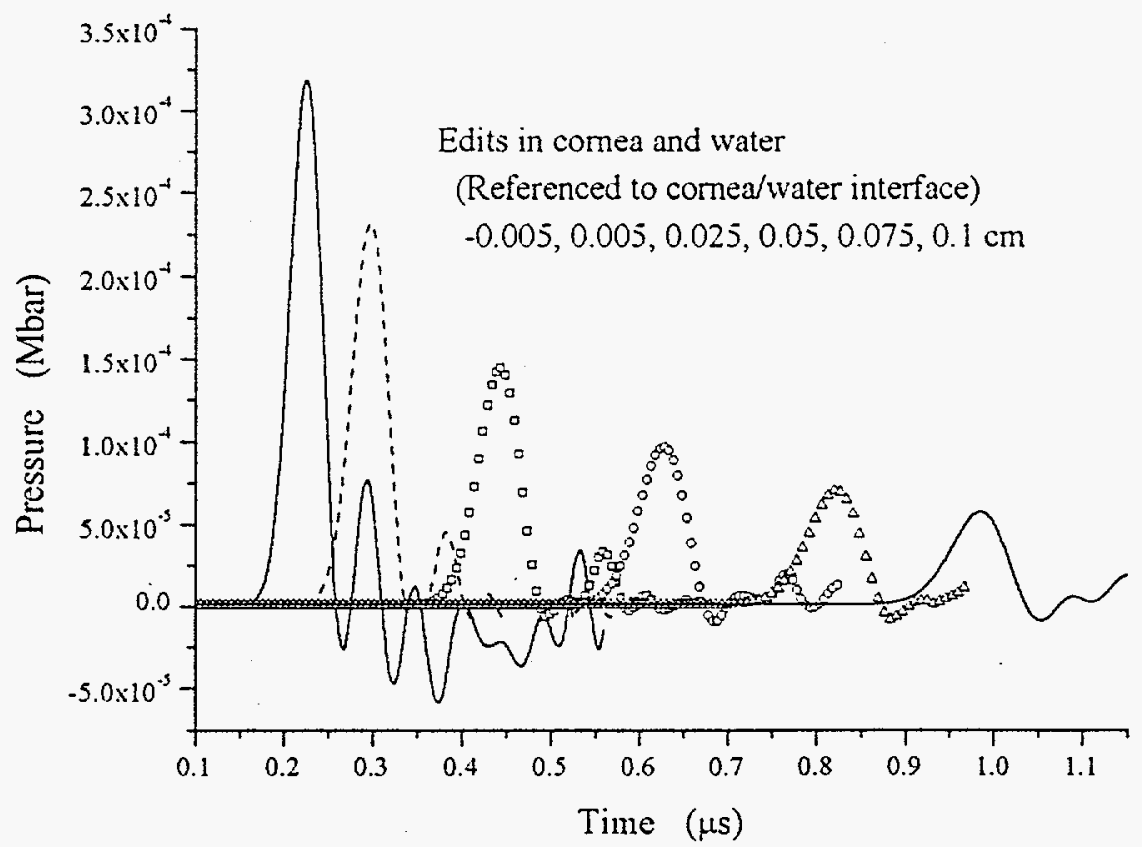

Figure 8: Evolution of the stress signals in comea (a) and water (b) for $Y_{0}=400$ bar and $G=200$ bar. 
(a)

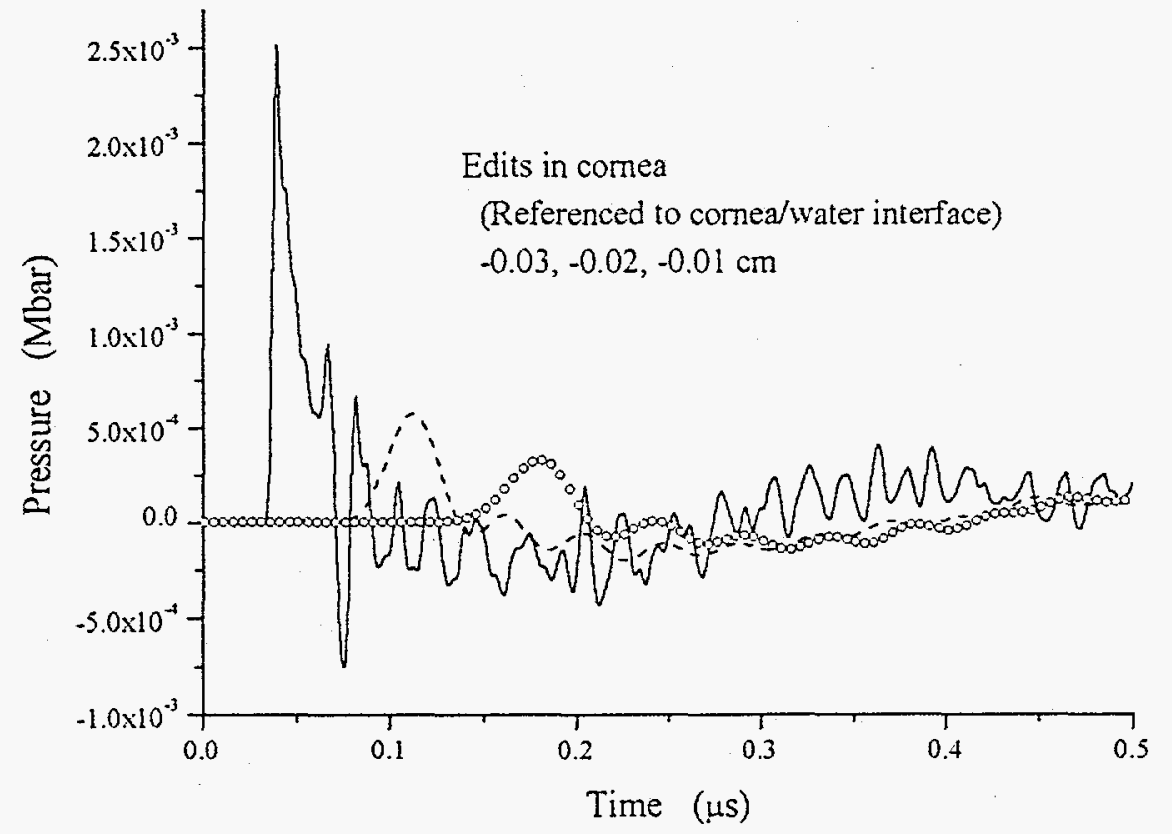

(b)

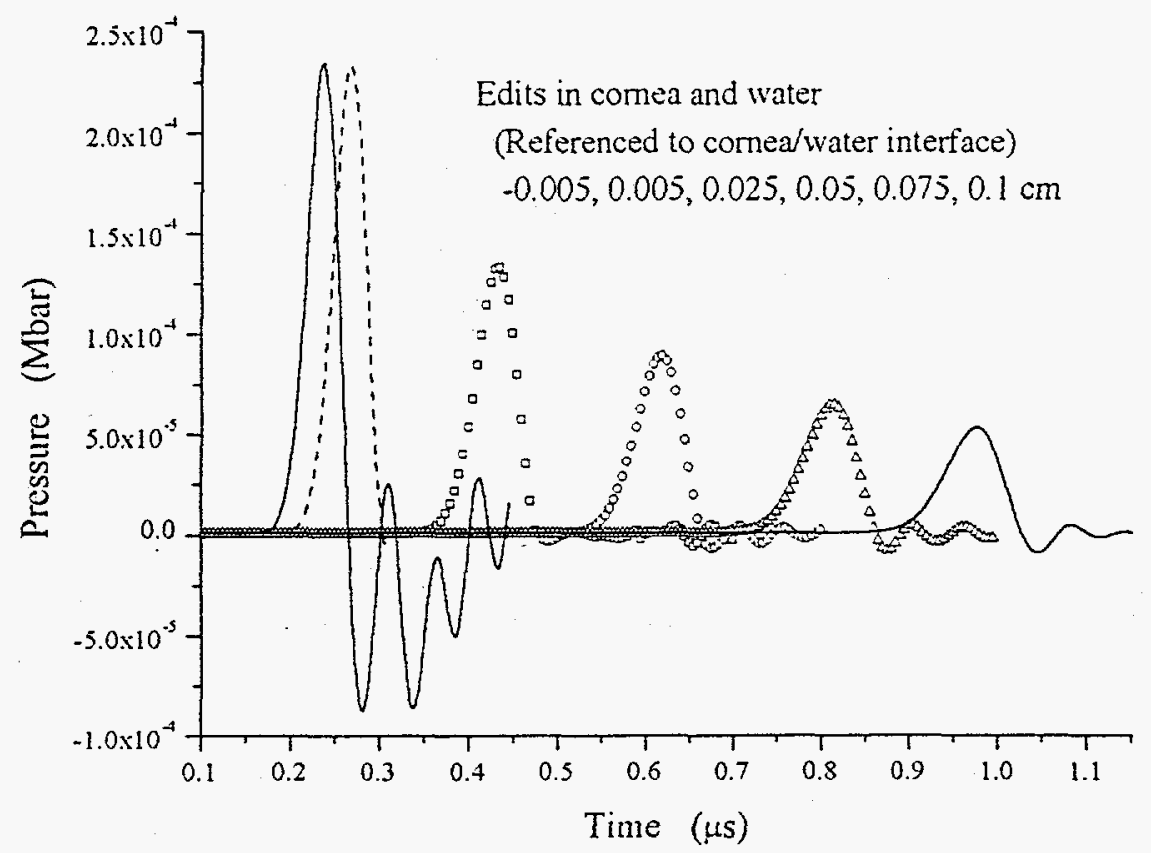

Figure 9: Evolution of the stress signals in comea (a) and water (b) for $Y_{0}=400 \mathrm{bar}, G=2000$ bar. 
(a)

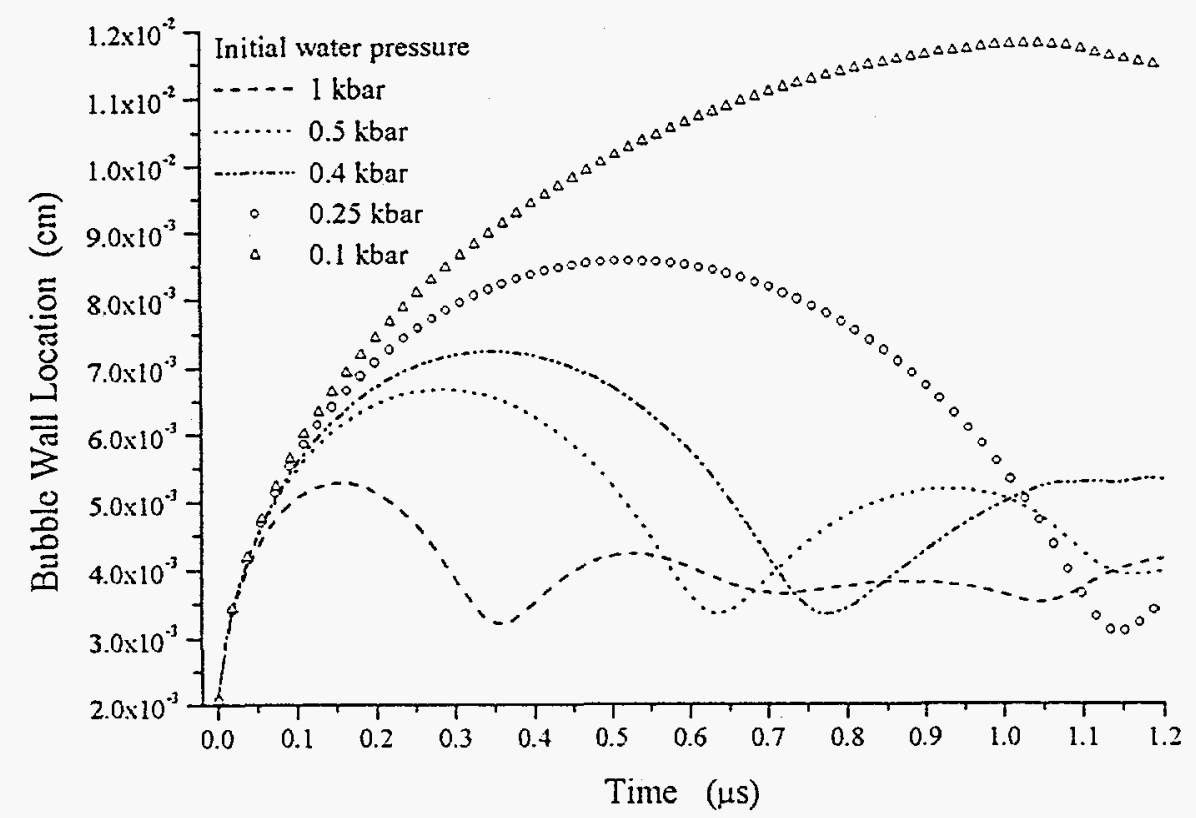

(b)

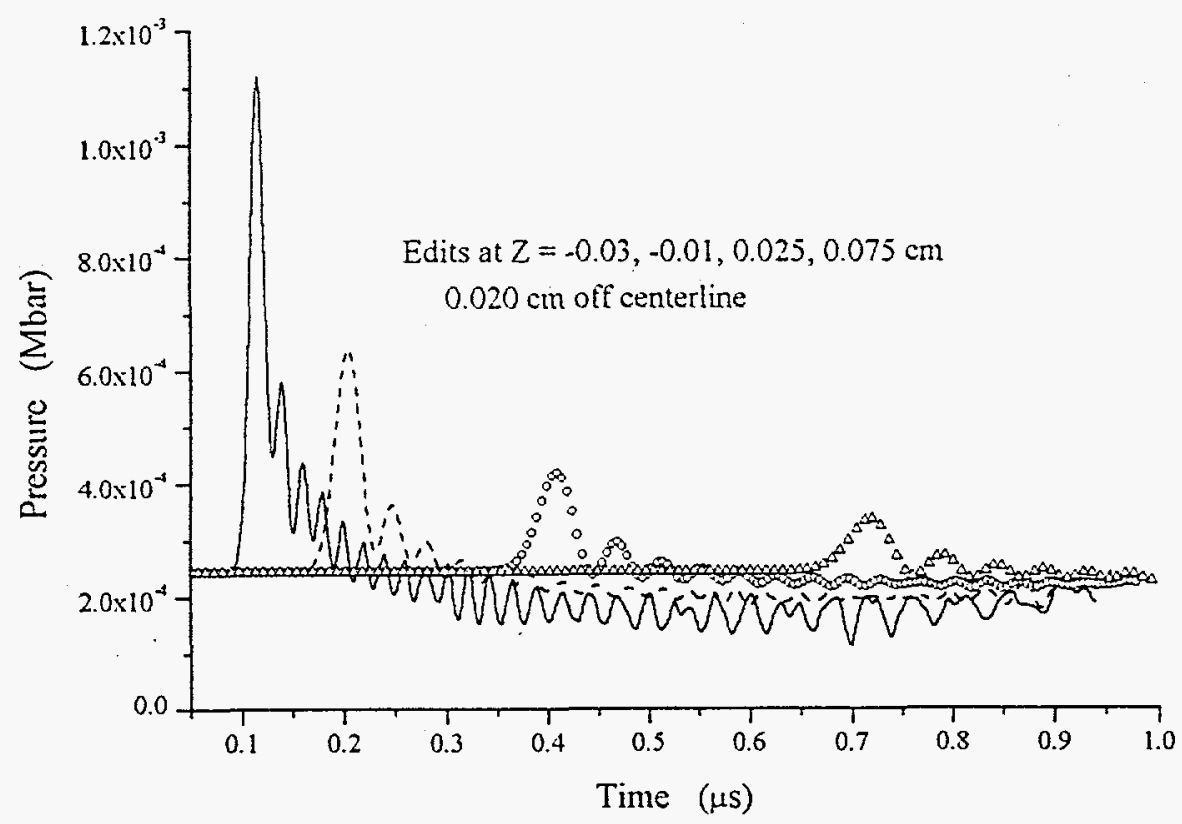

Figure 10: Bubble dynamics (a) and stress wave formation (b) in water at increased external pressure. The bubble dynamics was calculated for various values of $p_{\infty}$, and the stress wave evolution was analyzed for the $p_{\infty}$ value of 250 bar giving the best match between maximum bubble radius and experimentally observed bubble size. The compressive pulse is followed by a rarefaction wave, but not by tensile stress. 
(a)

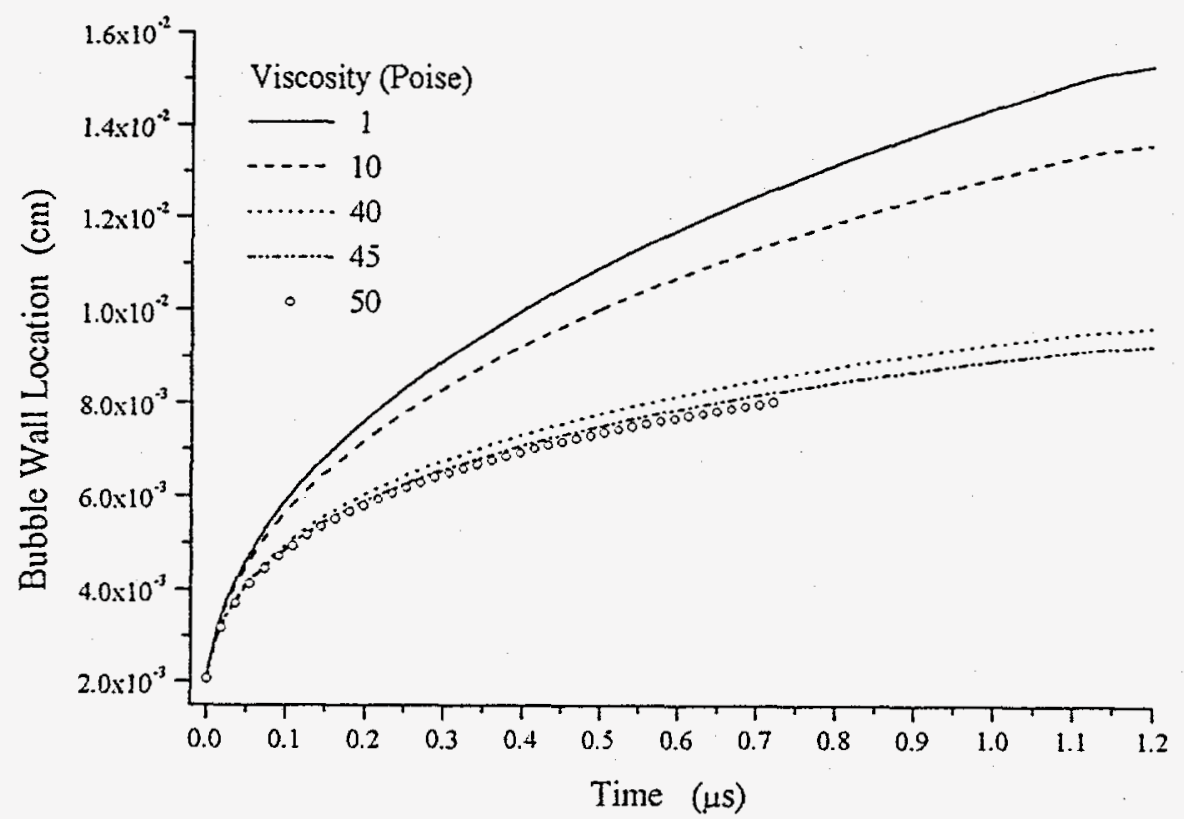

(b)

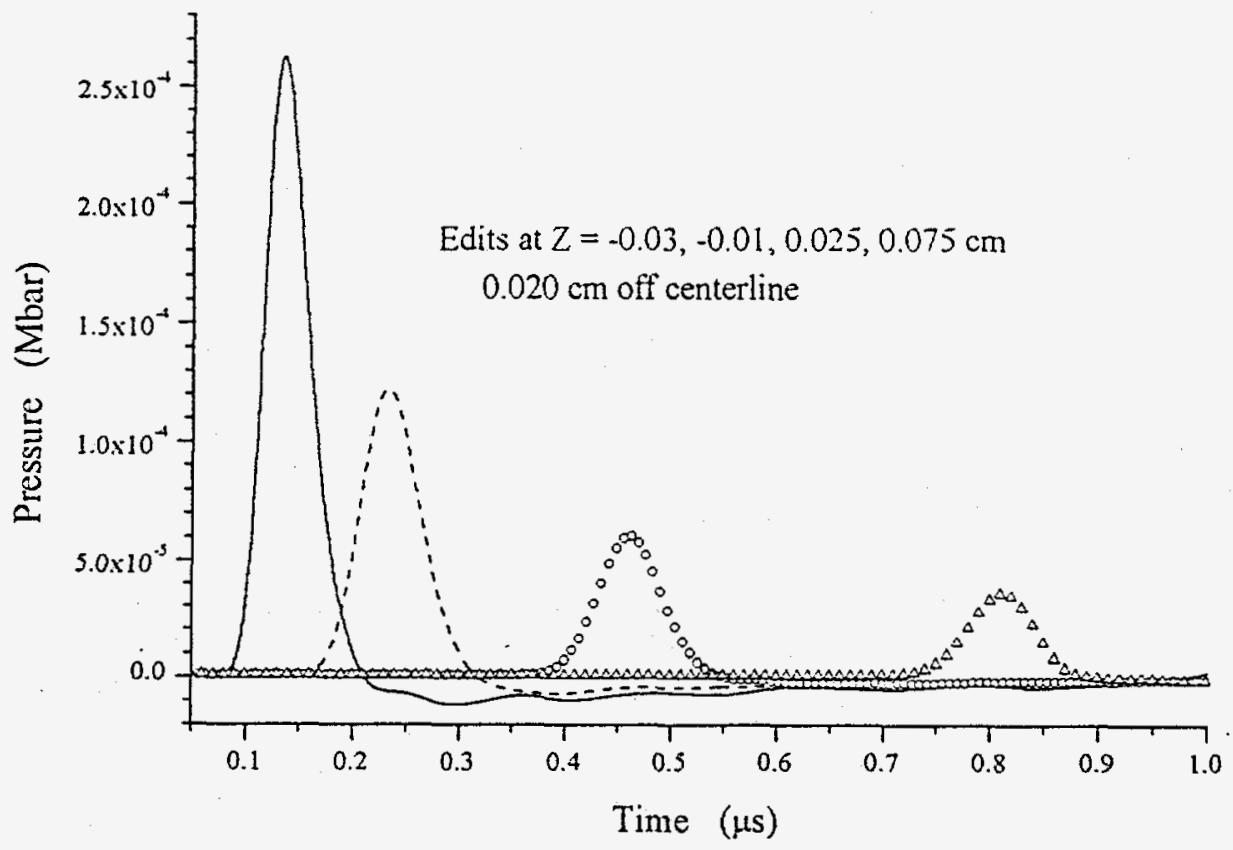

Figure 11: Bubble dynamics (a) and stress wave formation (b) in media with different viscosities but no elasticity. The stress wave evolution was analyzed for the viscosity value of $4.5 \mathrm{~Pa} \cdot \mathrm{s}$ ( 45 Poise) giving the best match between maximum bubble radius and experimentally observed bubble size. Note the small amplitudes of the compressive and tensile stress waves. 


\section{DISCUSSION}

\subsection{Origin of the Tensile Stress Wave}

A tensile stress wave was observed after optical breakdown in tissue, but not in water. To understand the origin of the tensile stress, we must therefore analyze the difference between the two cases.

The bubble oscillation is driven by the high pressure in the plasma produced during optical breakdown. In water, the bubble expands beyond its equilibrium radius due to inertial forces, and collapses again because of the hydrostatic pressure. When the bubble is maximally expanded, the internal pressure is very small. The reduced pressure inside the cavity is transmitted into the surrounding liquid and travels outward as a rarefaction wave, but there is no tensile stress (see Fig. 12).
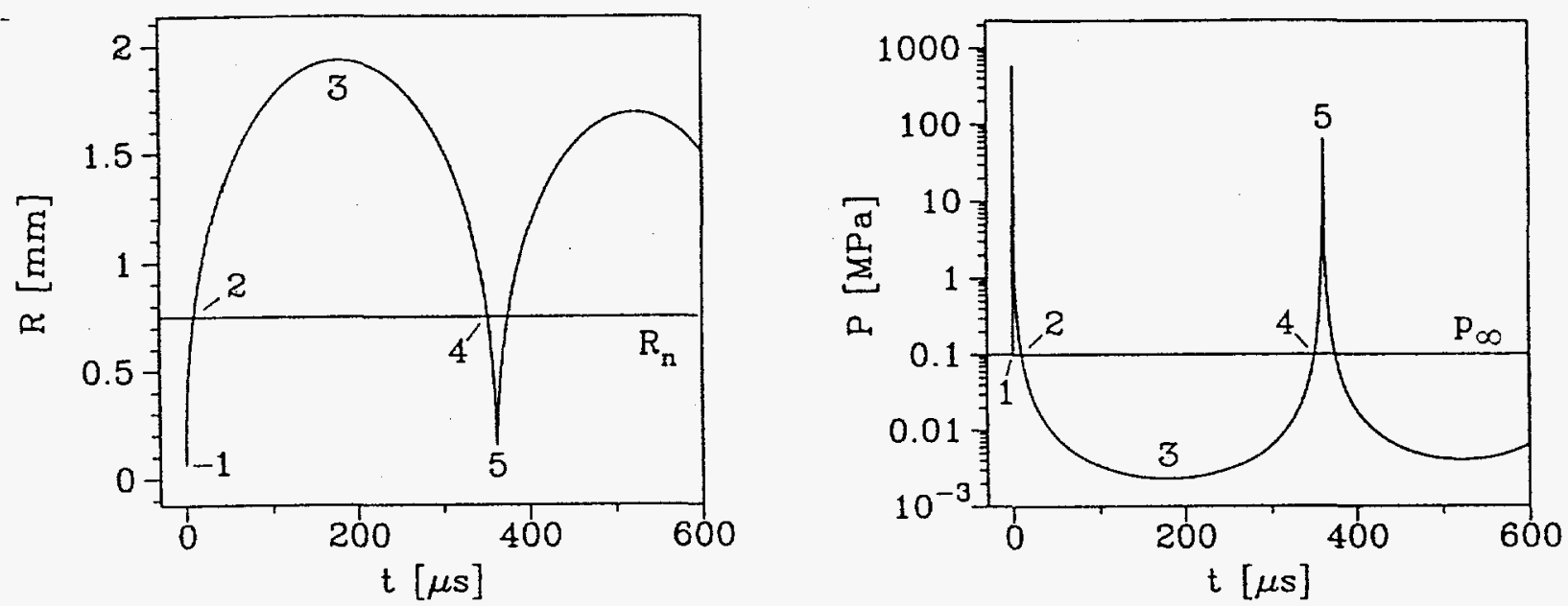

Figure 12: Evolution of the bubble radius and pressure at the bubble wall after optical breakdown in water. To cover the whole oscillation cycle with reasonable numerical etfort, the calculations were performed using the Gilmore model of bubble dynamics as described in Ref. [6]. $R_{\mathrm{n}}$ is the equilibrium radius of the bubble. The times 1 to 5 mark instants at which the impulse of the luid surrounding the bubble is either maximum (at $t_{2}$ and $t_{4}$ ) or zero (at $t_{1}, t_{3}$, and $t_{5}$ ) (see text).

When the breakdown takes place in tissue, the bubble oscillation period is shortened, because the elastic deformation of the tissue creates a restoring force which decelerates the outward motion of the bubble wall. Due to inertia, the bubble expands beyond the equilibrium radius where the bubble pressure balances the restoring elastic force plus the hydrostatic pressure. As a result, the restoring force increases further until the outward motion is finally stopped and the bubble starts to collapse. At this point, the restoring elastic force is much larger than the force exerted by the hydrostatic pressure, and the surrounding tissue is thus not only subjected to reduced pressure, but to tensile stress, that is, to a negative pressure.

As shown in Fig 11, (weak) tensile stress may also occur in a medium with high viscosity but no elasticity. That can be understood by looking at the temporal evolution of the impulse in the fluid around a spherical bubble. For unit area of the wave front, the impulse $I$ at location $r$ is given by ${ }^{33}$

$$
I\left(r, t^{\prime}\right)=\int_{0}^{t^{\prime}} \Delta p(r, t) d t,
$$

where $\Delta p$ denotes the difference between the actual pressure and the hydrostatic pressure. The medium in which the optical breakdown is generated has zero impulse before the breakdown and after the end of each bubble oscillation when the medium is at rest (times $I$ and 5 in Fig. 12). The impulse increases rapidly during the initial expansion phase when the bubble pressure is high and reaches ist maximum at time 2 when the driving force ceases. It diminishes during the 
following inertia-driven expansion and returns to zero when the bubble reaches ist maximum size and the fluid comes to rest (time 3). This corresponds to

$$
\int_{t_{1}}^{t_{2}} \Delta p(r, t) d t=-\int_{t_{2}}^{t_{3}} \Delta p(r, t) d t .
$$

A similar line of reasoning applies for the collapse phase: The impulse reaches a maximum with opposite sign at time 4 and returns to zero at time 5 (minimum bubble volume). This yields

$$
-\int_{t_{3}}^{t_{4}} \Delta p(r, t) d t=\int_{t_{4}}^{t_{5}} \Delta p(r, t) d t .
$$

In water, where the bubble oscillation is hardly influenced by viscous damping, the integrals in Eqs (3) and (4) have approximately the same value. In the presence of viscous damping, however, the impulse achieved during the collapse phase is much smaller than during bubble expansion. Consequently, no collapse pressure transient is observed, and the absolute value of the integral over the time interval $t_{3} \rightarrow t_{4}$ is much smaller than that over the interval $t_{2} \rightarrow t_{3}$.

In order to explain the origin of the tensile stress wave after breakdown in a tissue-like viscous medium, we concentrate on the bubble expansion phase. After the optical breakdown in water, interval $\left(t_{2} \rightarrow t_{3}\right)$ is much longer than interval $\left(t_{1} \rightarrow t_{2}\right)$, because the underpressure during $\left(t_{2} \rightarrow t_{3}\right)$ cannot be larger than the hydrostatic pressure of 1 bar, whereas the overpressure during $\left(t_{1} \rightarrow t_{2}\right)$ is in the kilobar range. In a medium with high viscosity, interval $\left(t_{1} \rightarrow t_{2}\right)$ is slightly prolonged due to the increased resistance of the medium surrounding the bubble and the absolute value of the integral over this interval is decreased due to the viscous damping, but the most pronounced effect is a shortening of the interval $\left(t_{2} \rightarrow t_{3}\right)$ as compared to the bubble expansion time in water. Eq. (3) can therefore only hold, if $\Delta p$ during interval $\left(t_{2} \rightarrow t_{3}\right)$ is larger than the hydrostatic pressure, i.e. if tensile stress is produced. The above argument applies not only to breakdown in a viscous medium, but also to a plastic-elastic deformation. In both cases, the occurrrence of tensile stress is linked to the amount of shortening of the bubble expansion time. However. at equal bubble expansion times, the amplitude of the tensile stress wave is larger in the elastic medium with less viscous damping, because the total impulse and, hence, $\Delta p$ are larger.

It is interesting to note that Delacretaz and Walsh ${ }^{34}$ observed no tensile stress when a cavitation bubble was produced in a tissue phantom by means of a free-running holmium laser pulse. When a bubble is produced by a free-running laser pulse, the life cycle of the bubble is comparable to the laser pulse duration, and the bubble growth is driven by the ongoing ablation/evaporation process. The ablation processes in tissue and water do not differ very much, and the stress wave formation is therefore also similar in both cases. After optical breakdown, however, the bubble expansion is driven by inertial forces during most of the bubble lifetime, because the laser pulse duration and the duration of the high initial bubble pressure are much shorter than the oscillation cycle of the bubble (Fig. 12). The response to the inertial forces differs strongly for tissue and water, and the stress wave formation is therefore strongly modified when the breakdown occurs in tissue.

\subsection{Effects of the Tensile Stress Wave}

The numerical simulations revealed that the maximum tensile stress after a $300 \mu \mathrm{J}, 30 \mathrm{ps} \mathrm{Nd:YAG} \mathrm{laser} \mathrm{pulse} \mathrm{focused}$ into the cornea is about -200 bar at $220 \mu \mathrm{m}$ distance from the source and -30 bar at the cornea-water interface (Fig. 8). The maximum bubble radius, however, is less than $100 \mu \mathrm{m}$ (Fig. 5). It is therefore possible that cellular damage due to tensile stresses is produced in regions which are not affected by the structural damage caused by the bubble expansion. Hamrick and Cleary ${ }^{35}$ showed that bipolar stress waves with a duration of 100 ns and an amplitude of 100 bar could cause breakage of the tobacco mosaic virus.

Experimentally, a tensile stress value of -1 bar was found at $10 \mathrm{~mm}$ distance from the laser focus (Fig. 6). That corresponds to -25 bar at the border between cornea and water if the amplitude of the stress transient decreases proportional to $1 / \mathrm{r}$ as observed for spherical compressive stress waves, and if the shape of the stress transient does not change during propagation. This value agrees well with the value of -30 bar obtained by numerical calculations. Because the cavitation threshold is about -10 bar, one should observe cavitation in the liquid surrounding the cornea specimen. Cavitation was, however, experimentally not detected. This fact is not yet fully understood. In a previous experiment, however, where the 
events during pulsed laser angioplasty were investigated in vitro with a silicone tube as a vessel model, we observed secondary cavitation on the exterior wall of the silicone tube ${ }^{18}$.

\subsection{Modeling of the Tissue Response}

London et al. ${ }^{17}$ assumed that the dynamics of laser-produced bubble in soft tissues may be modeled in a first order approximation by simply increasing the numerical value of the hydrostatic pressure to the value of the failure stress of the material. The calculations presented in Fig. 10 show, however, that this approach can explain neither the asymmetry between expansion and collapse phase of the bubble dynamics nor the tensile stress wave which were observed experimentally. In essence, this approach simply rescales the radius and growth-collapse cycle time of the Rayleigh model.

Modeling of the tissue as a fluid with high viscosity without consideration of an elastic response (Fig. 11) can explain the damping of the bubble oscillations and the lack of a pronounced collapse. However, because no elastic deformation is allowed, the viscous damping must be very high to restrict the bubble size to the experimentally observed value. The viscosity assumed was $4.5 \mathrm{~Pa} \cdot \mathrm{s}$ ( 45 Poise), i.e. 4500 times the viscosity of water. As a consequence of the strong viscous damping, the model predicts a 10 -fold reduction of the compressive peak of the acoustic signal and yields only a very small tensile stress component, both in contrast to the experimental observations (Fig. 6).

The assumption of an elastic-plastic material behavior chosen in this study is still a strong simplification of the actual viscoelastic tissue properties. It could, nevertheless, explain the generation of a tensile stress wave during optical breakdown in tissue. The key feature for the tensile stress generation is the elastic tissue response. This can be easily seen by setting the plastic flow stress $Y$ to very large values. In this case, the conditions resemble those of thermoelastic sound generation where bipolar stress waves are emitted from a point source in a homogeneous medium ${ }^{5}$. However, if the deformation was perfectly elastic, the bubble would collapse to a very small radius close to the initial plasma size. Consideration of the plastic deformation in the initial phase of bubble expansion is required to obtain a finite bubble size at the end of the oscillation cycles.

\subsection{Conclusions}

Stress wave generation and cavitation bubble dynamics after optical breakdown in tissue differ strongly from the dynamics in water. The bubble expansion time is considerably shortened, and this shortening leads to the formation of a tensile stress wave following the compressive pulse caused by the plasma expansion. Modeling the formation of the bipolar stress wave requires consideration of the elastic-plastic material response. Simple adjustments of individual parameters as viscosity, or ambient pressure do not yield satisfactory results.

Future experiments on tissue phantoms with isotropic properties may allow a more precise and definite comparison between simulations and experiments. The modeling needs to be refined to better portray the viscoelastic tissue properties, particularly the temporal and spatial dependence of the elastic and shear modulus on stresses and deformation rates, the viscosity during plastic deformation, and the anisotropic properties of tissues such as cornea.

\section{ACKNOWLEDGEMENTS}

We thank Dr. Enil Brujan for stimulating discussions on tissue material properties, Dr. Ulrich Parlitz for providing Fig. 12, and Edward J. Chapyak for numerical modeling advice. The work in the MLL was supported in part by the Deutsche Forschungsgemeinschaft. The work of LANL participants was supported in part by a CRADA between Los Alamos National Laboratory (DOE), Oregon Medical Laser Center, and Palomar Medical Technologies.

\section{REFERENCES}

1. J. B. Park, Biomaterials, An Introduction, Plenum Press, New York, 1979.

2. F. A. Duck, Physical Properties of Tissue, Academic Press, London, 1990.

3. R. S. Dingus, and R. J. Scammon, "Grüneisen-stress induced ablation of biological tissue", SPIE Proc. 1427, pp. $45-54,1991$ 
4. I. Itzkan, D. Albagli, M. L. Dark, L. T. Perelman, C. von Rosenberg, and M. Feld, "The thermoelastic basis of short pulsed laser ablation of biological tissue", Proc. Natl. Acad. Sci. 92, pp. 1960-1960, 1995

5. G. Paltauf and H. Schmidt-Kloiber, "Internal photomechanical fracture of spatially limited absorbers irradiated by short laser pulses", SPIE Proc. 3254, pp. 112-120, 1998

6. A. Vogel, S. Busch, and U. Parlitz, "Shock wave emission and cavitation bubble generation by picosecond and nanosecond optical breakdown in water", J. Am. Soc. Acoust. 100, pp. 148-165, 1996

7. R. J. Scammon, E. J. Chapyak, R. P. Godwin, and A. Vogel, "Simulations of shock waves and cavitation bubbles produced in water by nanosecond and picosecond laser pulses", SPIE Proc. 3254, pp. 264-275, 1998

8. A. Vogel, T. Günther, M. Asiyo-Vogel, R. Birngruber, "Factors determining the refractive effects of intrastromal photorefractive keratectomy with the picosecond laser", J. Cataract Refract. Surg. 23, pp. 1301-1310, 1997

9. R. M. Kurtz, C. Horvath, H. H. Liu, and Tibor Juhasz, "Optimal laser parameters for intrastromal corneal surgery", SPIE Proc. 3255, pp. 56-66, 1998

10. M. E. Glinsky, P. A. Amendt, D. S. Bailey, R. A. London, A. M. Rubenchik, and M. Strauss, "Extended Rayleigh model of bubble evolution with material strength compared to detailed dynamic simulations. SPIE Proc. 2975, pp. 318-334, 1997

11. E. J. Chapyak, and R. P. Godwin, "Physical mechanisms of importance to laser thrombolysis", SPIE Proc. 3245, pp. $12-18,1998$

12. A. Vogel, M. R. C. Capon, M. N. Asiyo-Vogel, and R. Birngruber, "Intraocular photodisruption with picosecond and nanosecond laser pulses: tissue effects in cornea, lens, and retina", Invest. Ophthalmol. Vis. Sci. 35, pp. 3032-3044, 1994

13. F. Docchio, C. Sacchi, and J. Marshall, "Experimental investigation of optical breakdown thresholds in ocular media under single puse irradiation with different pulse durations", Lasers Ophthalmol. 1, pp. 83-93, 1986

14. D. A. Hoeltzel, P. Altman, K. Buzerd, and K. I. Choe, "Strip extensiometry for comparison of the mechanical response of bovine, rabbit, and human corneas", Trans. ASWE. J. Biomech. Eng. 114, pp. 202-215, 1992

15. J. O. Hjortdal, "Regional elastic performance of the human cornea", J. Biomechanics 29, pp. 931-942, 1996

16. E. D. Jansen, T. Asshauer, M. Frenz, M. Moutamedi, G. Delacrétaz, and A. J. Welch, "Effect of pulse duration on bubble formation and laser induced pressure waves during holmium laser ablation", Lasers Surg. Med. 18, pp. $278-293,1996$

17. R. A. London, D. S. Bailey, P. Amendt, S. Visuri, and V. Esch, " A scaling model for laser-produced bubbles in soft tissue", SPIE Proc. 3254, pp. 256-263, 1998

18. A. Vogel, R. Engelhardt, U. Behnle, and U. Parlitz, "Minimization of cavitation effects in pulsed laser ablation illustrated on laser angioplasty", Appl. Phys. B 62, pp. 173-182, 1996

19. F. Soergel, S. Muecke, and W. Pechhold, "Comeal viscoelasticity spectra as a result from dynamic mechanical analysis", in J. Lass (Ed) Advances in Cornea Research: Selected Transactions of the World Corneal Congress, Plenum Publishing, New York 1997, pp. 239-254

20. E. Spörl, U. Genth, K. Schmalfuß, and T. Seiler, "Thermo-mechanical behavior of the cornea", Klin. Monatsbl. Augenheilk. 208, pp. 112-116, 1996 (in German)

21. B. Radt, "Mechanical Properties and Thermal Denaturation of Cornea", diploma thesis, University of Hamburg, and Medical Laser Center Lübeck, 1997 (in German)

22. D. M. Maurice, "The cornea and sclera", in: H. Davson (Ed.) The Eye, Vol 16, Academic Press, Orlando, 1984, pp. $1-158$

23. S. S. Chang, P. M. Pinsky, and D. V. Datye, "Inverse estimation of the in vivo mechanical properties of the cornea modeled as a transversely isotropic material", Invest. Ophthalmol. Vis. Sci. 36, No.4, p. S38, 1995

24. D. J. Benson, "Computational methods in Lagrangian and Eulerian hydrocodes", Computer methods in Applied Mechanics and Enegineering, 99, pp. 235-, 1992

25. A. Vogel, K. Nahen, D. Theisen, and J. Noack, "Plasma formation in water by picosecond and naosecond Nd:YAG laser pulses- Part I: Optical breakdown at threshold and superthreshold irradiance", IEEE J. Sel. Top. Quantum Electron. 2, pp. 847-860, 1996

26. K. Nahen and A. Vogel, "Plasma formation in water by picosecond and naosecond Nd:YAG laser pulses- Part II: Transmission, scattering and reflection", IEEE J. Sel. Top. Quantum Electron. 2, pp. 861-871, 1996

27. M. G. Sirotyuk, "Experimental investigations of ultrasonic cavitation", in L. D. Rozenberg (Ed) High Intensity Ultrasonic Fields, Plenum Press, New York 1971, pp. 263-346 
28. G. Paltauf, E. Reichel, and H. Schmidt-Kloiber, "Study of different ablation models by use of high-speed-sampling photography", SPIE Proc. 1646, pp. 343-352, 1992

29. S. L. Jaques, G. Gofstein, R. S. Dingus, "Laser flash photography of laser-induced spallation in liquid media", SPIE Proc. 1646, pp. 284-294, 1992

30. A. Vogel, M. N. Asiyo-Vogel, and R. Birngruber, "Investigations on intrastromal refractive surgery with picosecond Nd:YAG laser pulses", Ophthalmologe 91, pp. 655-662, 1994 (in German).

31. T. Juhasz, X. H. Hu, L, Turi, and Z. Bor, "Dynamics of shock waves and cavitation bubbles generated by picosecond laser pulses in corneal tissue and water", Lasers Surg. Med. 15, pp. 91-98, 1994

32. E. J. Chapyak, R. P. Godwin, S. A. Prahl, and H. Shangguan, "A comparison of numerical simulations and laboratory studies on laser thrombolysis" SPIE Proc. 2970, pp. 28-34, 1997

33. R. H. Cole, Undenvater Explosions, Princeton University Press, Princeton, 1948

34. G. Delacrétaz and J. T. Walsh, Jr, "Dynamic polariscopic imaging of laser-induced strain in a tissue phantom", Appl. Phys. Lett. 70, pp. 3510-3512, 1997

35. P. E. Hamrick and S. F. Cleary, "Breakage of tobacco mosaic virus by acoustic transients: a hydrodynamical model", J. Acoust. Soc. Am. 45, pp. 1-6, 1968 